\title{
Scaling of Bubble Growth in a Porous Medium
}

Topical Report

\author{
By \\ C. Satik, \\ Y. Yortsos, \\ and $\mathrm{X} . \mathrm{Li}$
}

July 1995

Work Performed Under Contract No. DE-FG22-93BC14899

\author{
Prepared for \\ U.S. Department of Energy \\ Assistant Secretary for Fossil Energy
}

Thomas Reid, Project Manager

Bartlesville Project Office

P.O. Box 1398

Bartlesville, OK 74005

Prepared by

University of Southern California

Dept. of Chemical Engineering

Los Angeles, CA 90089-1211 


\section{DISCLAIMER}

This report was prepared as an account of work sponsored by an agency of the United States Government. Neither the United States Government nor any agency thereof, nor any of their employees, makes any warranty, express or implied, or assumes any legal liability or responsibility for the accuracy, completeness, or usefulness of any information, apparatus, product, or process disclosed, or represents that its use would not infringe privately owned rights. Reference herein to any specific commercial product, process, or service by trade name, trademark, manufacturer, or otherwise does not necessarily constitute or imply its endorsement, recommendation, or favoring by the United States Government or any agency thereof. The views and opinions of authors expressed herein do not necessarily state or reflect those of the United States Government or any agency thereof. 


\section{DISCLAIMER}

Portions of this document may be illegible in electronic image products. Images are produced from the best available original document. 


\section{Contents}

List of Figures $\quad$ iv

1 INTRODUCTION 1

2 GROWTH PATTERNS

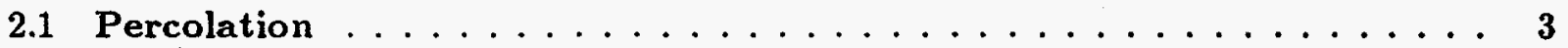

2.2 Viscous Fingering $\ldots \ldots \ldots \ldots \ldots \ldots \ldots \ldots \ldots \ldots \ldots \ldots \ldots \ldots \ldots \ldots \ldots \ldots$

3 RATES OF GROWTH $\quad 7$

4 CONCLUSIONS 9

$\begin{array}{lr}\text { REFERENCES } & 10\end{array}$ 


\section{List of Figures}

1 Experimental snapshots of gas cluster growth from carbonated water in a glass micromodel: (a) Large scale; (b) Pore scale sequence. . . . . . . . . . . . . . . . . . 2

2 Total net flux to a percolation cluster under quasi-static diffusion. The solid line is the theoretical slope $($ eqn. 1$) \ldots \ldots \ldots \ldots \ldots$

3 Percolation and viscous fingering boundaries from pore network simulations in a $50 \times 50$ square lattice. The solid line is a guide to the eye. . . . . . . . . . 7

4 Typical sequence of gas cluster growth under conditions of: (a) Capillary control $(C a=0.00001)$; (b) Viscous control $(C a=0.1) \ldots \ldots \ldots \ldots$ 


\section{INTRODUCTION}

Processes involving liquid-to-gas phase change in porous media are routinely encountered, for example in the recovery of oil, geothermal processes, nuclear waste disposal or enhanced heat transfer $[2,3,15,16]$. They involve diffusion (and convection) in the pore space, driven by an imposed supersaturation in pressure or temperature. Phase change proceeds by nucleation and phase growth. Depending on pore surface roughness, a number of nucleation centers exist, thus phase growth occurs from a multitude of clusters [18]. Contrary to growth in the bulk or in a Hele-Shaw cell $[8,13,14]$, however, growth patterns in porous media are disordered and not compact. As in immiscible displacements $[7,17]$, they reflect the underlying pore microstructure. The competition between multiple clusters is also different from the bulk. For example, cluster growth may be controlled by a combination of diffusion (e.g. Laplace equation in the quasi-static case) with percolation. Novel growth patterns are expected from this competition [11].

While multiple cluster growth is important, the simpler problem of single-bubble growth is still not well understood. In this section, we focus on the growth of a single bubble, subject to a fixed far-field supersaturation (e.g. by lowering the pressure in a supersaturated solution or by raising the temperature in a superheated liquid). Our emphasis is on deriving a scaling theory for growth at conditions of quasi-static diffusion, guided by recent experimental observations.

Visualization of bubble growth in model porous media was recently conducted $[9,10]$ using 2-D etched-glass micromodels. Fig. 1 shows a typical $\mathrm{CO}_{2}$ cluster evolving from carbonated water, initially saturated at $50 \mathrm{psi}$, the pressure of which was subsequently reduced to $14.7 \mathrm{psi}$. The non-compact nature of the cluster is apparent. The cluster grows in a manner similar to external drainage: gas-liquid interfaces are stable in the converging portion of a pore throat, but rapidly move to occupy an adjacent pore (in an event known as a "rheon"), once the capillary pressure barrier of that throat was exceeded. During the early stages, the growth occurs "one-site-at-a-time", which is a mode of interface advance typical of invasion percolation.

To model this problem, we developed a pore network simulation of multiple cluster growth ( $\mathrm{Li}$ and Yortsos [11]) and a scaling theory for single-bubble growth when mass transfer is diffusioncontrolled. This quasi-static limit is described below. We consider the initial conditions $C=$ $C_{0}, P_{l}=P_{0}$, and the far-field conditions $C=C_{0}, P_{l}=P_{\infty}<P_{0} \equiv K C_{0}$, where $C$ is solute concentration, $P_{l}$ is liquid pressure and $K$ is a solubility constant. Equivalently, the supersaturation 


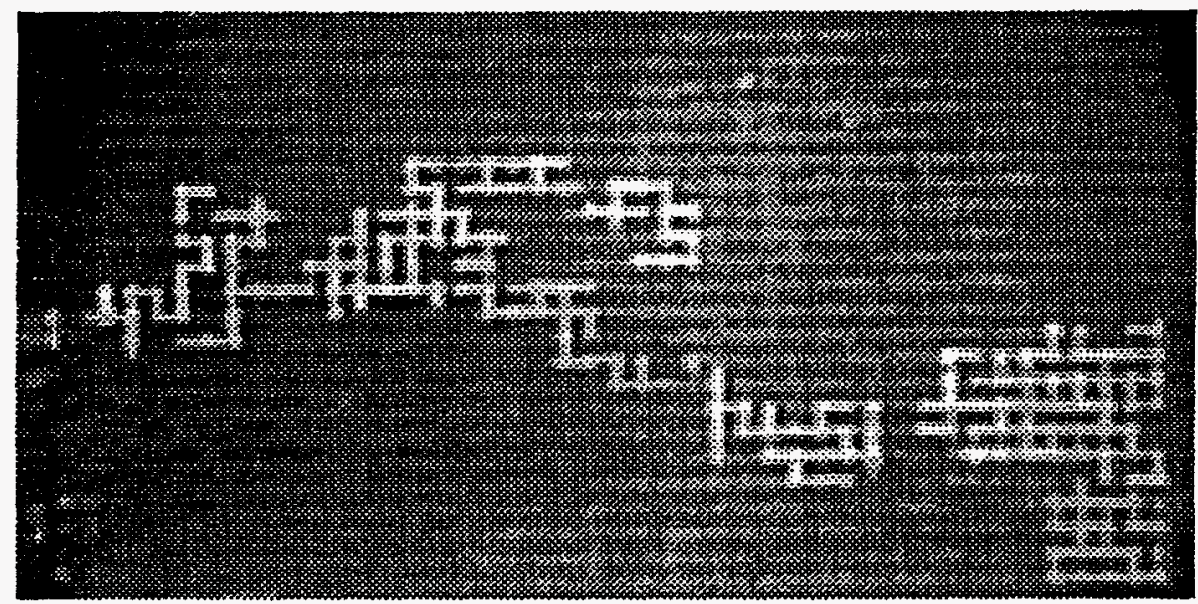

(a)
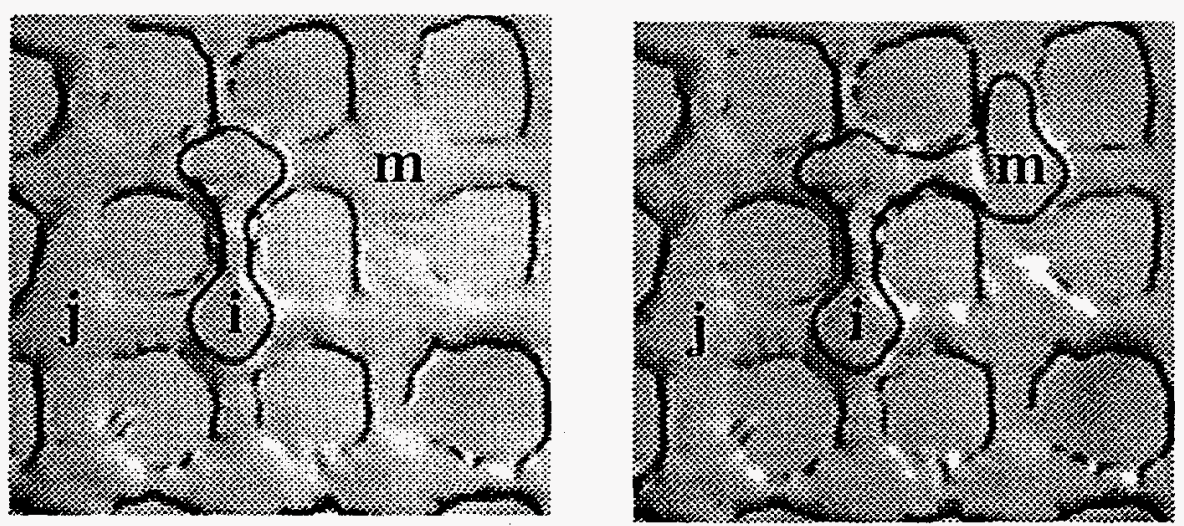

(b)

Figure 1: Experimental snapshots of gas cluster growth from carbonated water in a glass micromodel: (a) Large scale; (b) Pore scale sequence. 
can be expressed with the Jacols number, $J a=\frac{P_{0}-P_{\infty}}{P_{0}}$. At low supersaturations $(J a \ll 1)$ the concentration field in the liquid is quasi-static and satisfies the Laplace equation $D \nabla^{2} C=0$, where $D$ is the solute diffusivity. We consider a porous medium model in terms of an equivalent network of bonds (throats) and sites (pores). The gas-liquid interface consists of menisci residing on the cluster perimeter sites. We take the gas to be inviscid and ideal, such that $P_{g}$ is spatially uniform and $P_{g} V=n R T$, where $n$ is the number of moles in cluster of volume $V$. For simplicity, we take linear phase equilibria, $P_{g}=K C_{i}$, in all perimeter sites $i(i=1, N)$. Bubble growth is dictated by the net mass transfer rate, $\frac{d n}{d t}=\sum_{i, j} J_{i j}$, where the sum is over all perimeter sites $i$ and the liquid-occupied sites $j$ adjacent to them. The diffusive flux is $J_{i j}=D A_{i j} \frac{\left(C_{i}-C_{j}\right)}{l_{i j}}$, where the area $A_{i j}$ and the length $l_{i j}$ pertain to pore throat $i j$. Mass influx $\left(\frac{d n}{d t}>0\right)$ results into either pressurization $\left(d P_{g}>0\right)$ or bubble growth $(d V>0)$. Pressurization is necessary to overcome the capillary barrier of a perimeter bond, which occurs when the capillary pressure is sufficiently large, $P_{g}-P_{l, j}>\frac{2 \gamma}{r_{p, i j}}$, where $r_{p, i j}$ is the radius of the connecting bond and $\gamma$ the interfacial tension. Upon penetration, the interface advances and occupies site $j$. A measure of the driving force is the capillary number, $C a=\frac{J a D l^{*} \mu_{l}}{\gamma^{k}}$, where $l^{\star}$ is a characteristic lattice spacing, $k$ is permeability (which scales with the average throat size [4], $r_{p}^{\star}$, as $k \sim r_{p}^{\star 2}$ ) and the characteristic velocity $u^{*}$ is based on diffusion, $u^{\star}=\frac{J a D l^{\star}}{k}$. Note the difference with the conventional $C a$ in external displacements $[7,17]$. An additional relevant parameter is the solubility constant $\alpha=\frac{R T}{M_{w} K}$, where $M_{w}$ is the solute molecular weight.

To characterize bubble growth requires that the cluster pattern and its rate of growth be determined. Growth in the bulk or in an effective porous medium is compact and obeys the scaling $[8,13,14] R \sim t^{1 / 2}$. In a random porous medium we expect percolation at sufficiently small sizes and viscous fingering at larger sizes.

\section{GROWTH PATTERNS}

\subsection{Percolation}

The cluster will follow a percolation pattern, if perimeter bonds are invaded "one-at-a-time", such that the largest perimeter bond is always invaded first. These rules are the same with invasion percolation, except that here invasion occurs from an internal, rather than an external source. The 
following conditions must apply for percolation: (i) Immediately preceding and during pressurization, all interfaces reside in converging pore geometries. (ii) During filling, only "one-site-at-a-time" is invaded, the simultaneous penetration of another throat not being possible. Condition (i) is always statisfied, since liquid and gas pressures are spatially uniform (no flow) during pressurization. Condition (ii) depends on the viscous pressure drop. During filling of a partly occupied site $m$, the capillary pressure in the site is small, $P_{g} \sim P_{l, m}$. Simultaneous penetration at another location $l$ is not possible if $P_{g}-P_{l, l} \leq \frac{2 \gamma}{r_{p, l}}$, namely if the pressure difference between the two sites is small. At the percolation boundary, the pattern ceases being pure percolation due to the viscous pressure drop, which is in turn related to mass transfer. We shall denote by $R^{p}$ the cluster radius of gyration when this is reached and proceed for its estimation as follows.

During percolation, growth occurs from one site only (say $m$ ) (although mass transfer to the cluster is to all perimeter sites), thus the pressure field is set by the velocity of that site. The latter can be estimated from mass balance, $v_{m} \sim \frac{R T}{b P_{\infty}} \sum_{i, j} J_{i j}$ where $b$ denotes a length, $b=2 \pi h r_{s}^{\star}$ in 2-D and $b=4 \pi r_{s}^{\star 2}$ in $3-\mathrm{D}$, where $h$ is the thickness in 2-D and $r_{s}^{\star}$ a typical site radius. To calculate the mass flux, we must solve a problem of quasi-static diffusion in a Euclidean space, bounded internally by a fractal interface. Following Ref. [1], this flux equals the mean-field result

$$
\left.\sum_{i, j} J_{i j} \sim 2 \pi h D R^{p} \frac{\partial C}{\partial r}\right|_{r=R^{p}}=\frac{2 \pi h D\left(C_{0}-C_{i}\right)}{\ln \frac{R_{e}}{R^{r}}} \text { or } \sum_{i, j} J_{i j} \sim 4 \pi D\left(C_{0}-C_{i}\right) R^{p}
$$

in the two geometries, respectively, where $R_{e}$ denotes the outer boundary in 2-D. The velocity follows directly,

$$
v_{m} \sim\left(\frac{\alpha J a D}{r_{s}^{\star}}\right) \frac{1}{\ln \frac{R_{e}}{R^{r}}} \quad \text { or } \quad v_{m} \sim\left(\frac{\alpha J a D}{r_{s}^{\star}}\right)\left(\frac{R^{p}}{r_{s}^{\star}}\right)
$$

in the two geometries, respectively. For a conservative estimate of the percolation limit we calculate the pressure drop across a distance that scales with $R^{p}$. To describe viscous flow in a pore, we take Poiseuille's law. Then, the flow problem involves solving the Laplace equation subject to the velocity at site $m$ given from (2). As before, we will use a mean field approach (see also a related study [6]). We obtain,

$$
\Delta P_{v} \sim \frac{v_{m} \mu_{l} r_{s}^{\star}}{k} \ln \frac{R^{p}}{r_{s}^{\star}} \text { or } \quad \Delta P_{v} \sim \frac{v_{m} \mu_{l} r_{s}^{\star 2}}{k}\left(\frac{1}{r_{s}^{\star}}-\frac{1}{R^{p}}\right) \sim \frac{v_{m} \mu_{l} r_{s}^{\star}}{k}
$$


Then, substitution of (2) gives the final results

$$
\Delta P_{v} \sim \frac{J a D \alpha \mu_{l}}{k} \frac{\ln \frac{R^{p}}{r_{s}^{*}}}{\ln \frac{R_{e}}{R^{p}}} \quad \text { or } \quad \Delta P_{v} \sim \frac{J a D \alpha \mu_{l}}{k} \frac{R^{p}}{r_{s}^{*}}
$$

in the two geometries, respectively.

We next define the percolation limit by requesting [6] that variations, $\Delta S$, in the gas saturation due to penetration of more than one sites as a result of viscous forces are negligible: $\left|\frac{\Delta S}{S}\right|=\epsilon \ll 1$. From percolation, this is equivalently expressed as $\left|\frac{\Delta S}{S}\right| \sim\left(p-p_{c}\right)^{-1} \Delta p$, where $p$ is the percolation probability and $p_{c}$ the percolation threshold. In -capillary-controlled displacements, $p$ is related to the pore throat size distribution $\alpha_{p}\left(r_{p}\right)$ via $p=\int_{r_{p}}^{\infty} \alpha_{p}(r) d r$, hence $\Delta p \sim \alpha_{p}\left(r_{p}\right) \Delta r_{p} \sim \frac{\Delta r_{p}}{\sigma r_{p}}$, where $\sigma$ is a dimensionless measure of the variance of $\alpha_{p}\left(r_{p}\right)$. To relate $\Delta r_{p}$ to $\Delta P_{v}$, we note that variations in liquid pressure equal those in capillary pressure, $\Delta P_{v} \sim \Delta P_{c}$. The latter can be related to variations in the occupied pore sizes, $\Delta P_{c}=\frac{2 \gamma \Delta r_{p}}{r_{p}^{2}}$. Next, we identify the cluster extent with the cluster correlation length, thus $\frac{R^{r}}{l^{*}} \sim \xi \sim\left(p-p_{c}\right)^{-\nu}$, where $\nu$ is the correlation length exponent, equal to $\frac{4}{3}$ in $2-\mathrm{D}$ and to 0.88 in $3-\mathrm{D}$. Then, we replace variations in $p$ by variations in $r_{p}$, and substitute the above to find the results

$$
\left(\frac{R^{p}}{l^{\star}}\right)^{\frac{1}{\nu}}\left(\frac{\ln \frac{R^{p}}{r_{s}^{\star}}}{\ln \frac{R_{s}}{R^{p}}}\right) \frac{\alpha r_{p}^{\star} C \cdot a}{\sigma l^{\star}} \sim \epsilon \text { or }\left(\frac{R^{p}}{l^{\star}}\right)^{\frac{1}{\nu}+1} \frac{\alpha r_{p}^{\star} C a}{\sigma r_{s}^{\star}} \sim \epsilon
$$

in 2-D or 3-D geometries, respectively. Equation (5b) is analogous to the expression that delineates the percolation limit in external displacements [6]. Clearly, the radius at the percolation limit decreases as $C a$ increases, namely as $J a$ or $D$ increase.

\subsection{Viscous Fingering}

As the cluster size increases, the pattern eventually departs from percolation. To infer its characteristics, Li and Yortsos [8] performed a linear stability of the equivalent problem in an effective porous medium (or a Hele-Shaw cell). In the absence of capillarity, the growth posesses the MullinsSekerka instability of solidification $[5,12]$, which here also happens to coincide with the viscous fingering instability. As a result, we expect a limiting pattern of the DLA type at sufficiently large sizes. This was confirmed with numerical simulations (see below). To delineate the pattern boundary, $R^{v f}$, we proceed as in the previous. Now, however, we request that variations in the capillary 


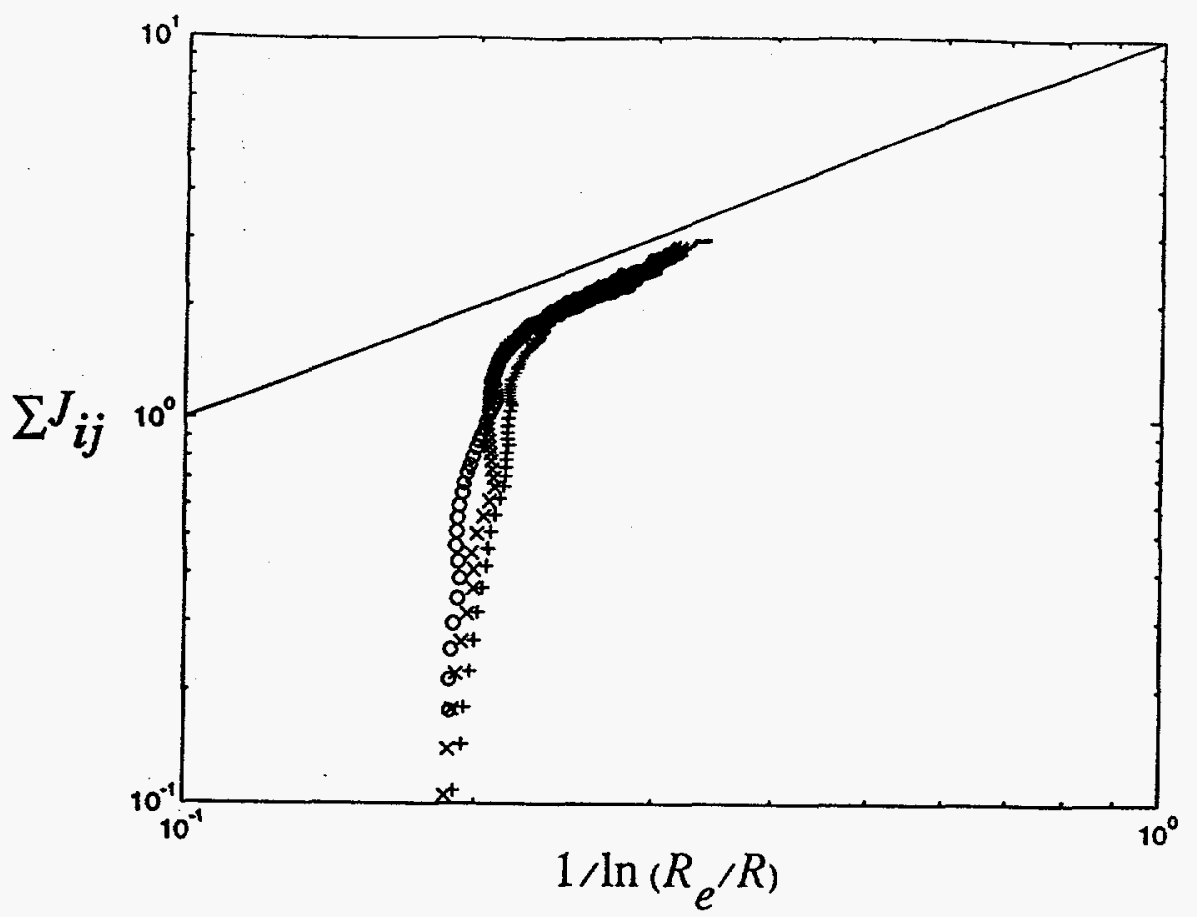

Figure 2: Total net flux to a percolation cluster under quasi-static diffusion. The solid line is the theoretical slope (eqn. 1).

pressure are small compared to the viscous pressure drop between adjacent sites, $\Delta P_{c} \ll \Delta P_{v}$. For the latter, we take

$$
\Delta P_{v} \sim \frac{v_{m} \mu_{l} r_{s}^{\star}}{k} \ln 2 \quad \text { or } \quad \Delta P_{v} \sim \frac{v_{m} \mu_{l} r_{s}^{\star}}{2 k}
$$

and after substitution of $v_{m}$, we obtain

$$
\left(\frac{1}{\ln \frac{R_{\star}}{R^{v f}}}\right)\left(\frac{\alpha C a}{\sigma}\right) \sim \epsilon^{-1} \quad \text { or } \quad\left(\frac{R^{v f}}{l^{\star}}\right)\left(\frac{\alpha C a l^{\star}}{\sigma r_{s}^{\star}}\right) \sim \epsilon^{-1}
$$

in the two geometries, respectively. Large values of $C a$ result in promoting a pure DLA growth pattern at smaller cluster sizes.

We used the numerical simulator in 2-D geometries (Li and Yortsos [11]), to test the validity of the two limits. Simulations were performed in square lattices of variable sizes, but not larger than $50 \times 50$, due to computational limitations. First, the mean-field results for quasi-static mass transfer were tested. Fig. 2 shows a plot of the total net mass flux to a percolation cluster as a function of the cluster size. Good agreement with the theory is found provided that the computational domain is large (here equal to $200 \times 200$ ) and the cluster size sufficiently large, but not too large for the 


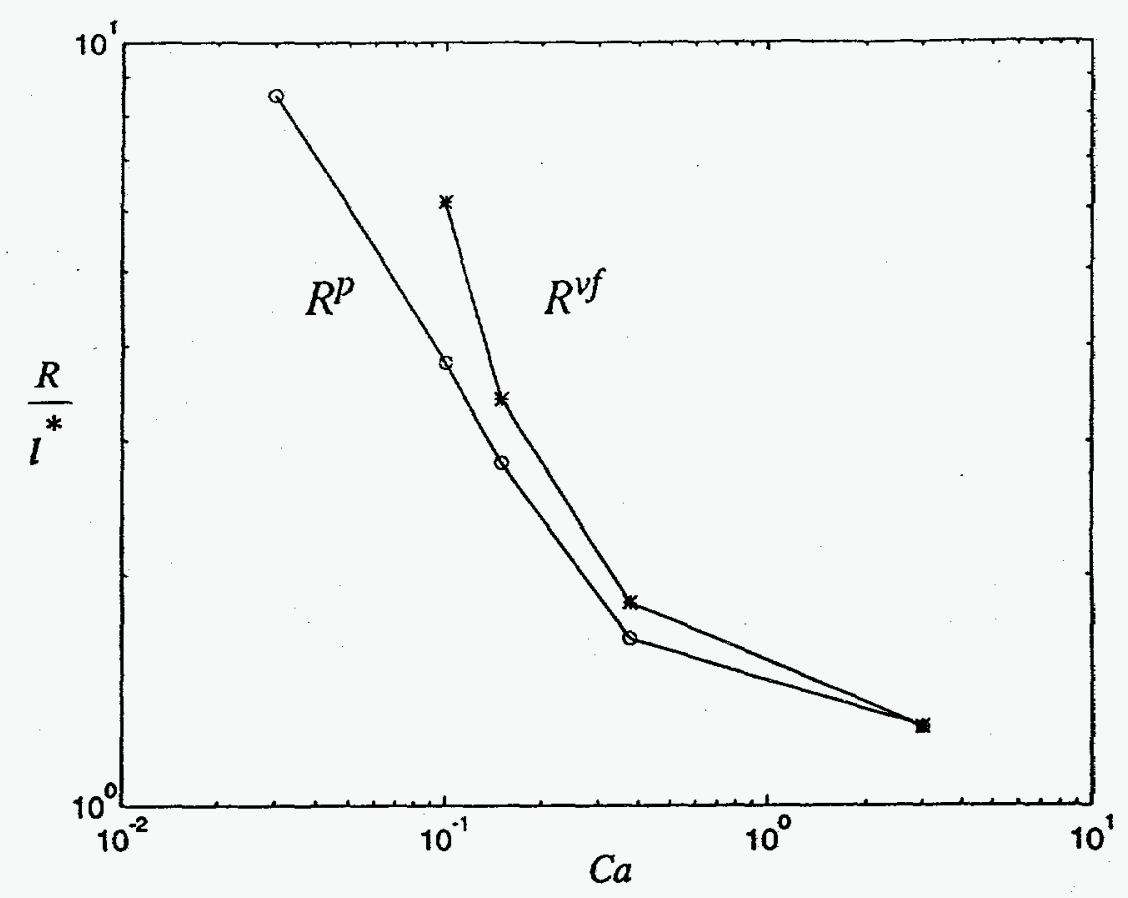

Figure 3: Percolation and viscous fingering boundaries from pore network simulations in a $50 \times 50$ square lattice. The solid line is a guide to the eye.

boundary to affect the radial symmetry. Plotted in Fig. 3 are numerical results for $R^{p}$ and $R^{v f}$, where only quasi-static diffusion was considered (the simulator can also account for convection and transients [11]). The qualitative trend of the results is consistent with the theory. However, due to the small size of the computational domajn ( $50 \times 50)$, which gives rise to substantial finite-size effects, a quantitative agreement is difficult to be ascertained. The cluster must be large enough for meaningful percolation statistics, yet small enough compared to the outer boundary for the meanfield theories to be valid. We expect a better agreement as the size increases to at least $200 \times 200$ (compare with Fig. 2). The transition from percolation to DLA is illustrated in Fig. 4, which illustrates cluster growth under conditions of percolation (Fig. 4a) or viscous (Fig. 4b) control.

\section{RATES OF GROWTH}

From the above, rates of bubble growth can be calculated. If $N_{g}$ is the number of sites occupied by the gas cluster and $V_{s}$ the average site volume, we have $V_{s} \frac{d N_{g}}{d t}=v_{m} b$, where $N_{g}$ is related to the radius $R_{g}$ via $N_{g} \sim\left(\frac{R_{g}}{f^{*}}\right)^{D_{f}}$ and $D_{f}$ is the fractal dimension of the cluster. Substitution from 

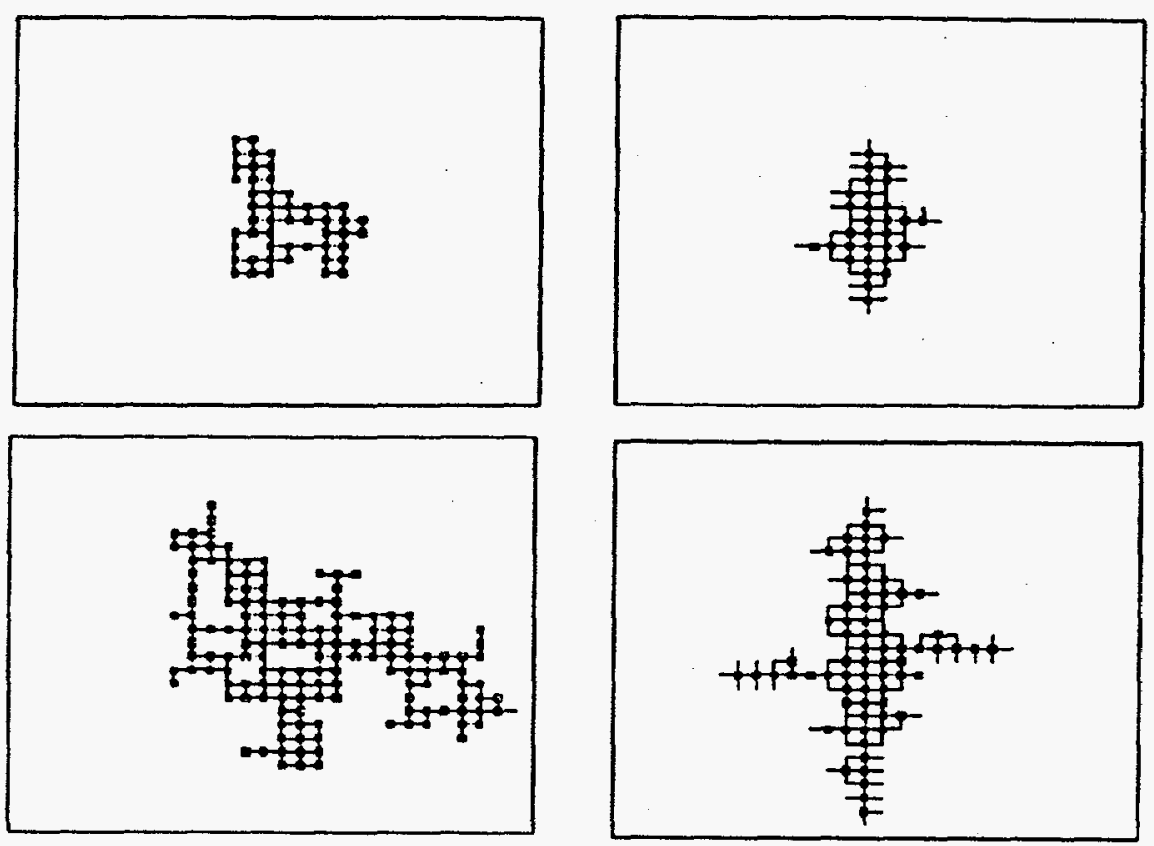

(a) Capillary Control

(b) Viscous Control

Figure 4: Typical sequence of gas cluster growth under conditions of: (a) Capillary control ( $\mathrm{Ca}=$ $0.00001)$; (b) Viscous control $(C a=0.1)$. 
(2) yields the results

$$
\left(\frac{R_{g}}{l^{\star}}\right)^{D_{f}}\left[1+D_{f} \ln \frac{R_{e}}{R_{g}}\right] \sim \frac{\alpha b J a D D_{f}}{V_{s} r_{s}^{\star}} t \text { or }\left(\frac{R_{g}}{r}\right)^{D_{f}-1} \sim \frac{\left(D_{f}-1\right)}{D_{f}} \frac{\alpha b l^{\star} J a D}{V_{s} r_{s}^{\star 2}} t
$$

in the two geometries, respectively. The two scalings should be contrasted to the classical for growth in the bulk, $R_{g}^{2} \sim t$. As a result of its ramified structure, the cluster grows faster than in its effective medium analogue (for example, $R_{g} \sim t^{\frac{2}{3}}$, for the 3-D case in either the percolation or the viscous fingering limits).

All these scalings rely on the assumption of quasi-static mass transfer. For growth in the bulk this is equivalent to the condition $J a \ll 1$. In the general case, however, this condition becomes [11] $J a t^{\frac{3-D_{f}}{D_{f}^{-1}}} \ll 1$. Thus, contrary to the compact cluster $\left(D_{f}=3\right)$ the validity of the quasi-static approximation in fractal patterns $\left(D_{f}<3\right)$ is time-dependent. To check its validity when $R^{p}$ and $R^{v f}$ are reached we substitute in (5) and (7) the results from (8) to obtain $J a^{1-\frac{\nu}{\nu+1}\left(3-D_{f}\right)} \ll 1$ and $J a^{1-\frac{D_{f}\left(3-D_{f}\right)}{D_{f}-1}} \ll 1$, respectively. Since $D_{f} \sim 2.5$ in both patterns, the two conditions are still equivalent to the condition for bulk growth, $J a \ll 1$.

\section{CONCLUSIONS}

We conclude that during single bubble growth in a porous medium the following regimes develop in succession: a short duration early-time regime, where finite size effects dominate, the growth is still compact and the effective medium scaling applies; a percolation regime $\left(R_{g} \leq R^{p}\right)$; a transition to a viscous fingering regime $\left(R^{p} \leq R_{g} \leq R^{v f}\right)$; and a DLA regime $\left(R^{v f} \leq R_{g}\right)$. In the absence of convection, the corresponding rates of growth are different than the classical. Effects of convection are under study. 


\section{References}

[1] M. E. Cates and T. A. Witten. Physical Rev. A, 35:1809-1824, 1987.

[2] A. De Swaan. J. Pet. Tech., page 907, 1981.

[3] E. B. Jr. Hunt and V. M. R. Berry. AICHEJ, page 560, 1956.

[4] A. J. Katz and Thompson A. H. Phys. Rev. B, 34:8175, 1987.

[5] J. S. Langer. Rev. Mod. Phys., 52:1, 1980.

[6] R. Lenormand. Proc. R. Soc. Lond., A423:159, 1989.

[7] R. Lenormand. J. Phys.: Condens. Matter, 2:SA79, 1990.

[8] X. Li and Y. C. Yortsos. Submitted to Phys. Fluids A, 1993.

[9] X. Li and Y. C. Yortsos. Paper SPE 22589 presented at the 1991 Fall Meeting of the SPE, Dallas, TX, Oct. 6-9 1991.

[10] X. Li and Y. C. Yortsos. Submitted to AIChEJ, 1993.

[11] X. Li and Y. C. Yortsos. Submitted to Chem. Eng. Sci., 1993.

[12] P. Pelce (ed). The Dynamics of Curved Fronts. Academic Pres, 1988.

[13] M.S. Plesset and A. Prosperetti. Ann. Rev. Fluid Mech., 9:145-185, 1977.

[14] L.E. Scriven. Chem. Eng. Sci., 10:1-13, 1959.

[15] C.H. Sondergeld and L. Turcotte. J. Geophys. Res., 82:2045-2053, 1977.

[16] J. R. Thome. Enhanced Boiling Heat Transfer. Hemisphere Publishing Co., 1990.

[17] D. Wilkinson and J. F. Willemsen. J. Phys. A, 16:3365-3376, 1983.

[18] E. Yousfi, C. Zarcone, S. Bories, and R. C. Lenormand. R. Acad. Sci. Paris, 313, Series II:1093, 1991. 